WHC-EP--0394-1

DE9 1007160

\title{
Ground Water Maps of the Hanford Site
}

\author{
G. L. Kasza \\ S. F. Harris \\ M. J. Hartman \\ Date Published \\ December 1990
}

Prepared for the U.S. Department of Energy

Office of Environmental Restoration and

Waste Management

(2) Wostingthouse P.O. Box 1970

Hestord Company Richland, Washington 99352

Hantord Operations and Engineering Contractor tor the

U.S. Depertment of Energy under Contract DE-AC06-87RL10950 
WHC-EP-0394-1

\section{INTRODUCTION}

This report presents the results of the June 1990, ground wate level measurement program at the 100 Areas and the 200 Areas of the Hanford Site (Figure 1). The water levels beneath these areas are measured regularly on a semiannual basis and the data received are used to produce the following set of maps for public release. For clarity, the locating prefixes have been omitted from all well numbers shown on the maps. Wells in the 100 Areas have the prefix 199; wells in the 200 Areas have the prefix 299, and the wells outside these areas have the prefix 699.

Ground Water Maps of the Hanford Site is prepared by the Geosciences Group, Environmental Division, Westinghouse Hanford Company, for the U.S. Department of Energy, Richland Operations Office.

\section{AREAS}

This report location comprises the various 100 Area reactor facilities and the surrounding land south of the Columbia River and north of Gable Mountain and Gable Butte. Reactor operations have ceased at all of the facilities and environmental restoration activities are in progress. The maps for this area are Figure 2, 100 Areas Depth-to-Water Map, and Figura 3, 100 Areas Water Table Map. The maps are based on June 1990 field measurements from wells that meet the following criteria: (a) wells north of Hanford Coordinate $\mathrm{N} 60000$; (b) wells completed in the unconsolidated sediments; and (c) wells completed within $100 \mathrm{ft}$ of the average water table. Data were averaged where there was more than one measurement in June. A representative set of wells from the 100 Areas were chosen where there are heavy concentrations of wells (e.g., 100-N and 100-H areas).

An interesting feature on the 100 Areas water table map is the area of high water levels just north of Gable Mountain. This feature has been present on water table maps of the Hanford Site for many years (e.g., Bierschenk 1959). Three of the wells that have the most "unusual" data (699-66-39, 699-66-38, and 699-69-38) were resurveyed by Kaiser Engineers Hanford on July 3,1990. No significant errors in the database elevations were discovered; thus, the water level elevations appear to be valid. The driller's log for well 699-66-39 is less complete; it shows sand and gravel at the surface, and primarily clay from 60 to $90 \mathrm{ft}$. Well 699-69-38 is a dug well and there is no record of its geology. It is possible that these wells monitor a zone of perched water, trapped in and on top of the finegrained sediments. However, the water table map in this document was drawn as if these wells express the true water table.

Another unusua? feature of the June 1990 water table map is the trough in the water table near the Columbia River in the eastern part of the 100 Areas. River levels were extremely high in May and June. A reversed gradient existed near the river, causing water levels in the aquifer adjacent to the river to rise. A reversed gradient undoubltedly also existed immediately adjacent to the river in the western part of the 100 Areas; however, it was apparently a much smaller feature and is not evident in 
WHC - EP-0394-1

data from wells near the river (e.g., wells 199-N-8S and 190-0-3). This feature is less prominent because the average ground water gradient towards the river is generally steeper in the western part of the 100 Areas than in the eastern part. Water level data used to construct the 100 Areas maps are listed in Table 1.

\section{AREAS}

This report location consists of the 200 East and 200 West areas and the surrounding vicinity on the Hanford Site south of Hanford Coordinate N60000. The semiannual measurement of water levels in the 200 Areas is performed on the more than 180 selected wells that comprise the Operational Groundwater Monitoring Network*. The Operational Groundwater Monitoring Network provides water level measurement for the determination of hydraulic gradient and water quality sampling of the ground water beneath and surrounding the active and inactive chemical processing and waste management facilities in the 200 Areas. Water level data from several non-Network wells are included on the maps to provide supplemental information for ease of interpretation.

The 200 Areas set of maps consists of a 200 Areas Depth-to-Water Map (Figure 4), a 200 Areas Water Table Map (Figure 5), and a map comparing the potentiometric surface of the Rattlesnake Ridge confined aquifer to the water table of the unconfined aquifer (Figure 6 ). Water level data used to construct these maps is presented in Table 2.

\section{REFERENCE}

Bierschenk, W. H., 1959, Aquifer Characteristics and Ground-Water Movement at Hanford, HW-6060l, General Electric Company, Hanford Atomic Products Operation, Richl and, Washington.

*Operational Groundwater Monitoring at the Hanford Site 1988, December 1989, Westinghouse Hanford Company, Richland, Washington. 


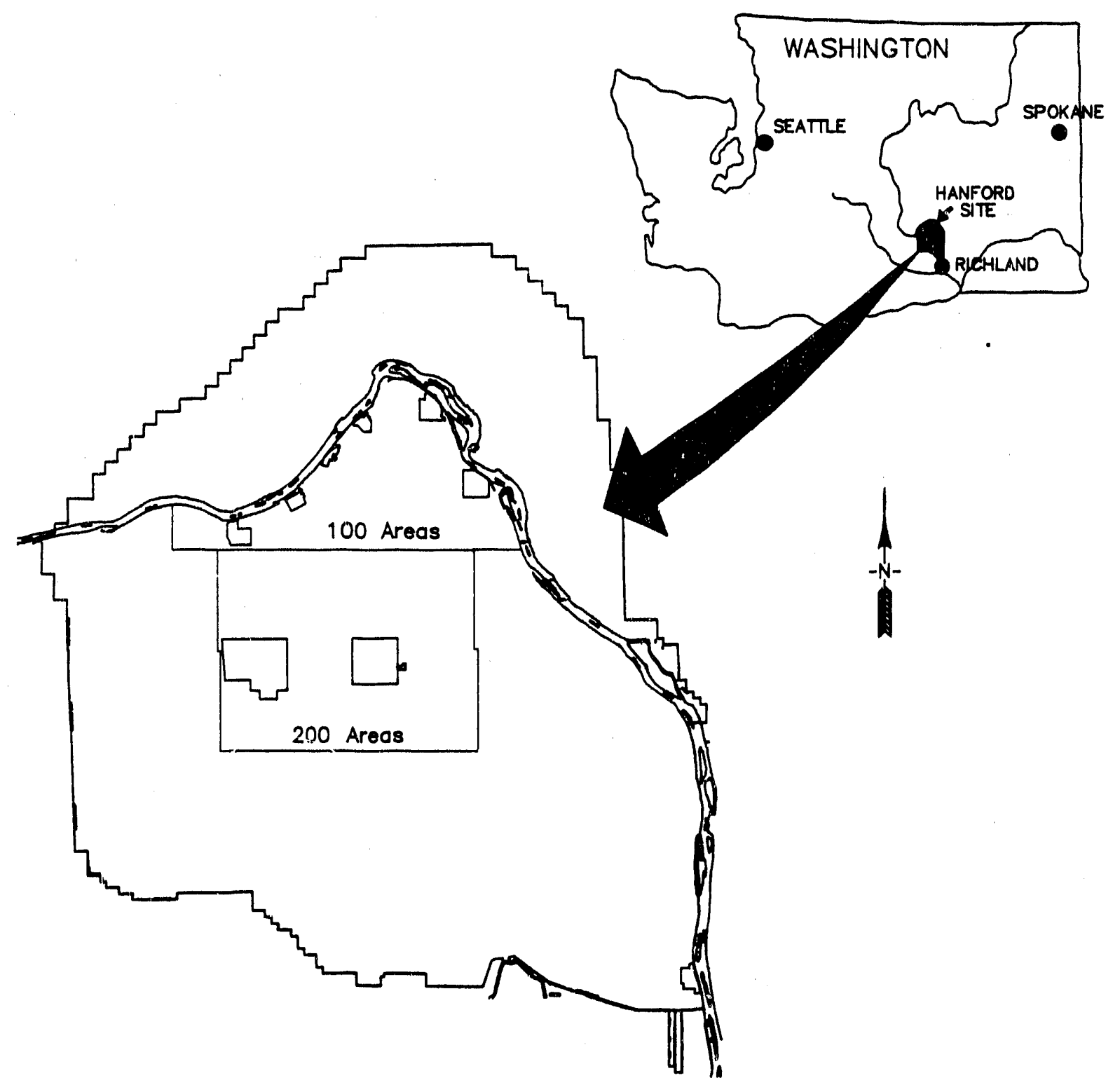

Figure 1. Hanford Index Map 


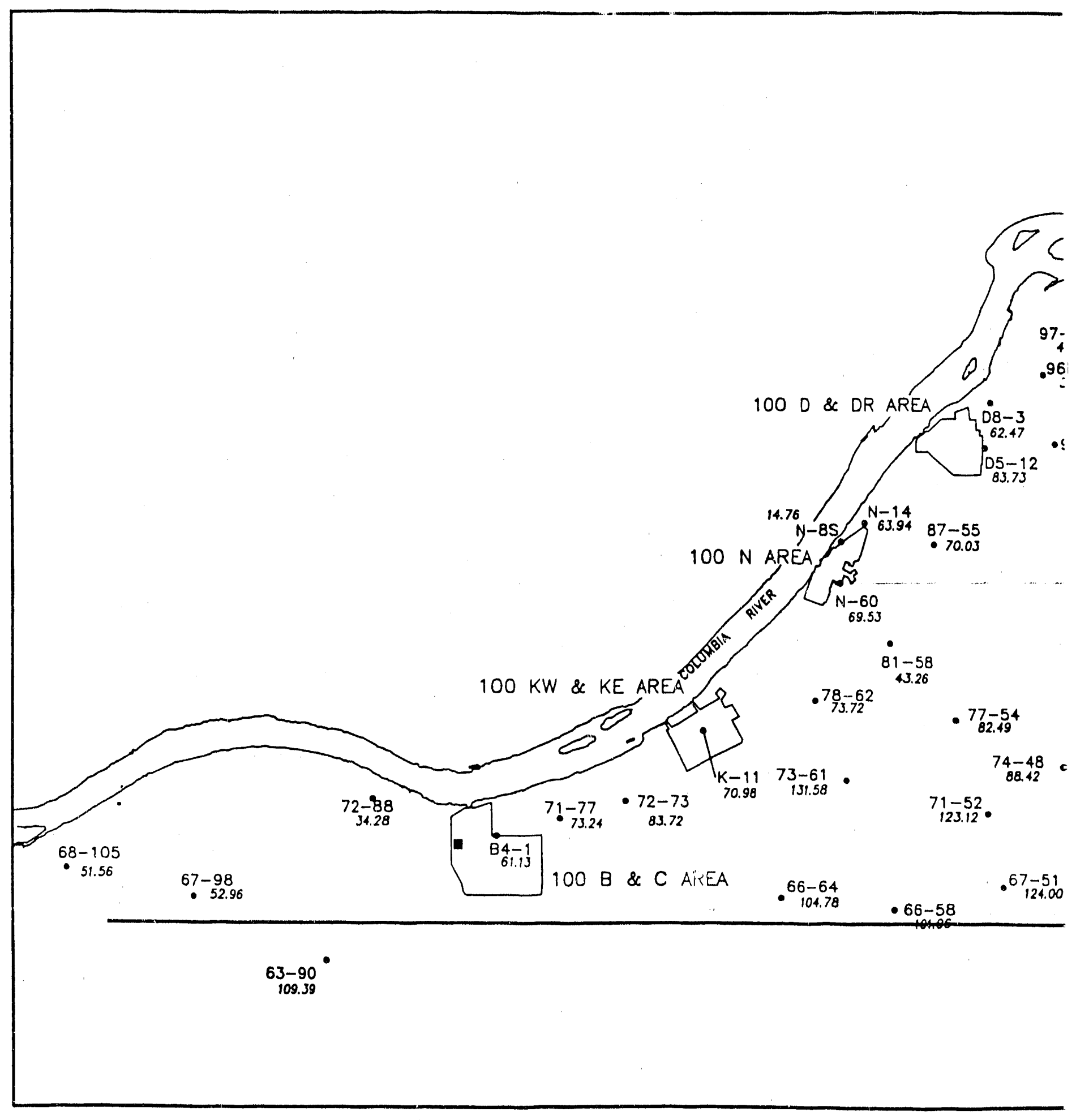




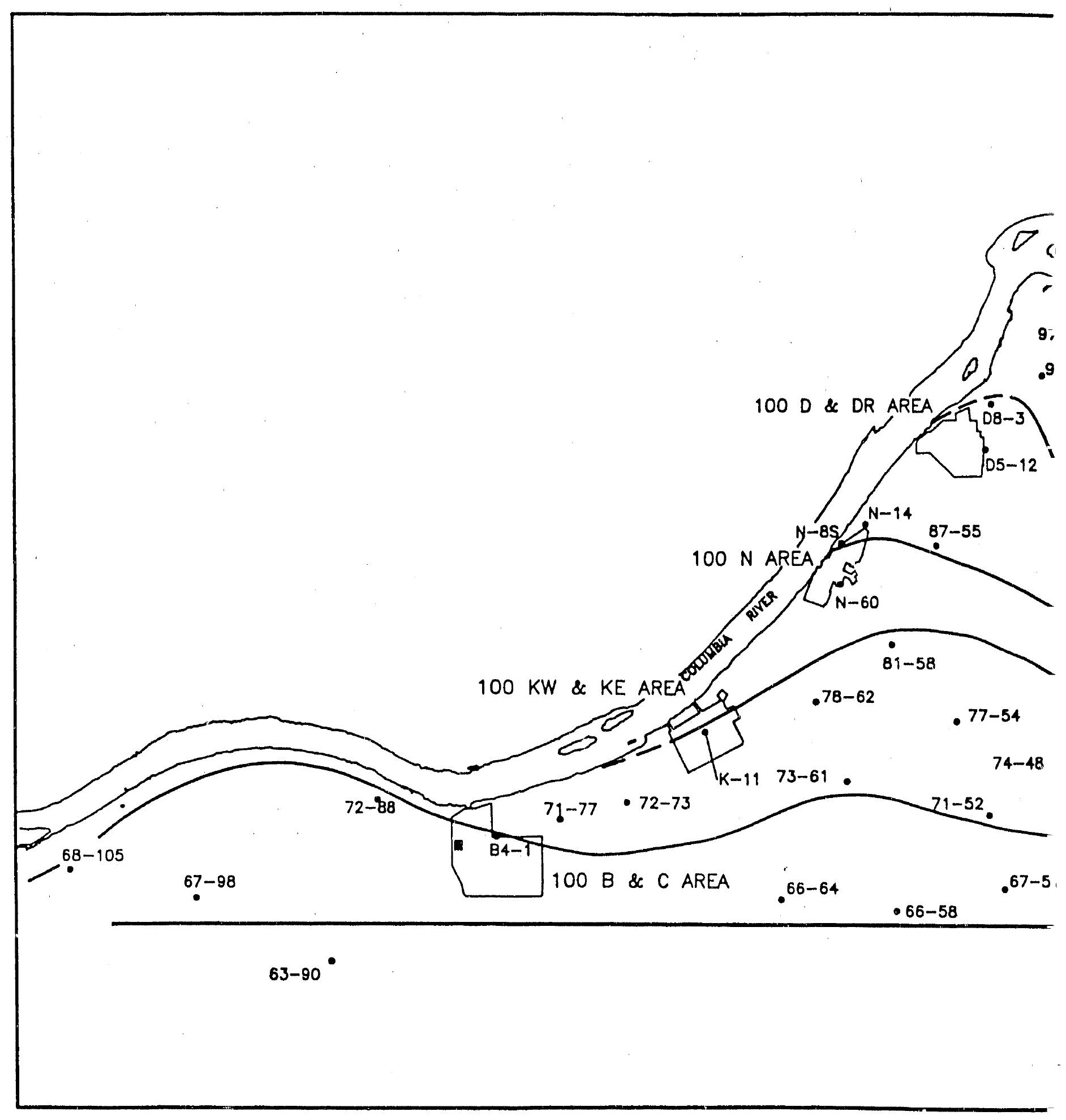




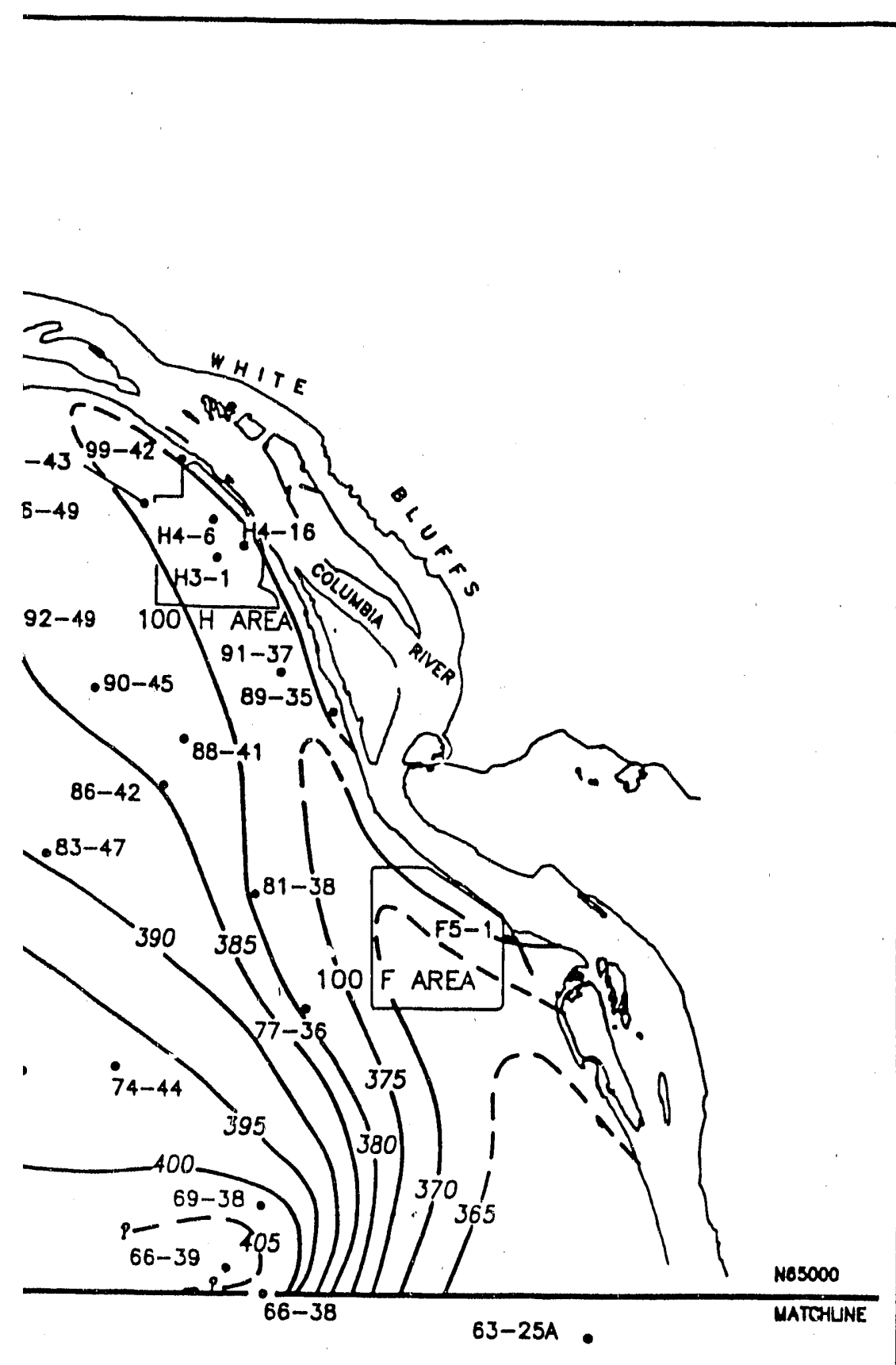

Figure 3

\section{Areas \\ Water Table Map June 1880}
390 - Water toble contours in feet above mean sea level.
7-40 Dato points used to prepare map.
The 100 Areas water table map has been prepared by the Geosciences Group. Environmental Division. Westinghouse Hanford Company.

Note: To convert to metric, multiply elevation (ft) by 0.3048 to obtain elevation (m).
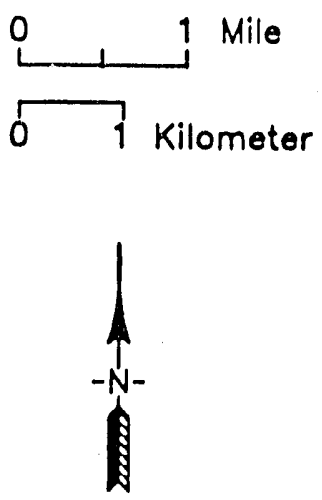
Table 1. June 1990 Water Level Measurement Data, 100 Areas. (Sheet 1 of 4)

We11

Depth to

Elevation, ft above msl

Water, ft Adjusted Casing Water Level

\begin{tabular}{|c|c|c|c|}
\hline $\begin{array}{l}199-B 4-01 \\
199-D 2-05 \\
199-D 5-12 \\
199-D 8-03 \\
199-F 5-01\end{array}$ & $\begin{array}{l}61.13 \\
73.71 \\
83.73 \\
62.47 \\
30.44\end{array}$ & $\begin{array}{l}461.80 \\
460.87 \\
469.67 \\
449.06 \\
406.56\end{array}$ & $\begin{array}{l}400.67 \\
387.16 \\
385.94 \\
386.59 \\
376.12\end{array}$ \\
\hline $\begin{array}{l}199-H 3-01 \\
199-H 3-02 A \\
199-H 4-10[N] \\
199-H 4-11[N] \\
199-H 4-12 A\end{array}$ & $\begin{array}{l}44.30 \\
39.88 \\
23.31 \\
36.10 \\
32.51\end{array}$ & $\begin{array}{l}421.48 \\
417.83 \\
404.44 \\
416.84 \\
413.50\end{array}$ & $\begin{array}{l}377.18 \\
377.96 \\
381.14 \\
380.74 \\
380.99\end{array}$ \\
\hline $\begin{array}{l}199-\mathrm{H} 4-13[\mathrm{~N}] \\
199-\mathrm{H} 4-14 \\
199-\mathrm{H} 4-15 A[\mathrm{~N}] \\
199-\mathrm{H} 4-16 \\
199-\mathrm{H} 4-17\end{array}$ & $\begin{array}{l}37.69 \\
42.66 \\
26.10 \\
16.63 \\
39.31\end{array}$ & $\begin{array}{l}418.20 \\
420.59 \\
407.21 \\
424.23 \\
419.09\end{array}$ & $\begin{array}{l}380.52 \\
377.93 \\
381.11 \\
377.60 \\
379.79\end{array}$ \\
\hline $\begin{array}{l}199-\mathrm{H} 4-18[\mathrm{~N}] \\
199-\mathrm{H} 4-03[\mathrm{~N}] \\
199-\mathrm{H} 4-04[\mathrm{~N}] \\
199-\mathrm{H} 4-05[\mathrm{~N}] \\
199-\mathrm{H} 4-06\end{array}$ & $\begin{array}{l}42.86 \\
41.00 \\
32.77 \\
35.77 \\
41.57\end{array}$ & $\begin{array}{l}421.82 \\
420.29 \\
413.70 \\
416.21 \\
419.58\end{array}$ & $\begin{array}{l}378.97 \\
379.29 \\
380.93 \\
380.44 \\
378.01\end{array}$ \\
\hline $\begin{array}{l}199-\mathrm{H} 4-07 \\
199-\mathrm{H} 4-08[\mathrm{~N}] \\
199-\mathrm{H} 4-09[\mathrm{~N}] \\
199-\mathrm{K}-11 \\
199-\mathrm{N}-14\end{array}$ & $\begin{array}{l}42.07 \\
40.94 \\
38.51 \\
70.98 \\
63.94\end{array}$ & $\begin{array}{l}420.59 \\
420.00 \\
418.08 \\
467.66 \\
453.15\end{array}$ & $\begin{array}{l}378.52 \\
379.07 \\
379.57 \\
396.68 \\
389.21\end{array}$ \\
\hline $\begin{array}{l}199-N-16 \\
199-N-17[N] \\
199-N-18[N] \\
199-N-19[N] \\
199-N-02[N]\end{array}$ & $\begin{array}{l}64.82 \\
69.85 \\
67.45 \\
62.73 \\
68.27\end{array}$ & $\begin{array}{l}456.70 \\
461.20 \\
458.50 \\
453.90 \\
459.83\end{array}$ & $\begin{array}{l}391.88 \\
391.35 \\
391.05 \\
391.17 \\
391.56\end{array}$ \\
\hline $\begin{array}{l}199-N-20[N] \\
199-N-21[N] \\
199-N-23[N] \\
199-N-24[N]\end{array}$ & $\begin{array}{l}64.38 \\
65.58 \\
65.11 \\
41.41\end{array}$ & $\begin{array}{l}455.90 \\
457.00 \\
456.30 \\
432.50\end{array}$ & $\begin{array}{l}391.52 \\
391.42 \\
391.19 \\
391.09\end{array}$ \\
\hline
\end{tabular}

LEGEND:

[N] Not locaied on maps to improve visual clarity.

[Q] Questionable data - not included on map. 
WHC -EP-0394-1

Table 1. June 1990 Water Level Measurement Data, 100 Arteas. (Sheet 2 of 4 )

We11

Depth to

Elevation, ft above ms 1 Water, ft Adjusted Casing Water Level

$199-N-25[N]$

$199-N-26[N]$ $199-N-27[N]$ $199-\mathrm{N}-28[\mathrm{~N}]$ $199-N-29$

$199-N-03[N]$

$199-\mathrm{N}-31[\mathrm{~N}]$ $199-N-32[N]$ $199-N-33[N]$ $199-N-34[N]$ $199-N-36[N]$

199-N-37 [N] $199-N-39[N]$ $199-\mathrm{N}-04[\mathrm{~N}]$ $199-N-40[N]$ 199-N-41

$199-\mathrm{N}-42[\mathrm{~N}]$ $199-N-44[N]$ $199-N-51$ [N] 199-N-52 $199-N-53[N]$

$199-\mathrm{N}-54[\mathrm{~N}]$ $199-N-55[N]$ $199-N-56[N]$ 199-N-57 [N] $199-N-58[N]$

$199-N-59[N]$

199-N-06 [N] $199-\mathrm{N}-60[\mathrm{~N}]$ $199-N-62[N]$ $199-N-63[N]$

$199-\mathrm{N}-64[\mathrm{~N}]$ $199-N-65[N]$
35.28

64.58

57.08

71.78

72.73

68.33

70.82

70.21

68.16

67.60

67.41

64.81

63.38

67.03

65.92

67.70

65.24

69.69

72.72

71.48

71.10

65.75

66.08

66.68

65.67

70.49

67.17

68.42

69.53

71.12

74.48

62.57

64.58
425.80

455.80

449.08

464.24

465.25

459.45

462.63

462.08

459.87

459.63

458.97

456.12

454.31

458.73

456.35

457.59

455.14

460.70

462.18

463.70

461.76

457.51

457.85

458.09

457.76

462.88

459.53

460.97

461.94

463.59

466.70

454.63

456.44
390.52

391.22

391.92

392.46

392.52

391.12

391.81

391.87

391.71

392.03

391.56

391.31

390.93

391.70

390.43

389.89

389.90

391.01

389.46

392.22

390.66

391.76

391.77

391.41

392.09

392.39

392.36

392.55

392.41

392.47

392.22

392.06

391.86

LEGEND:

[N] Not located on maps to improve visual clarity.

[Q] Questionable data - not included on map. 
Table 1. June 1990 Water Level Measurement Data, 100 Areas. (Sheet 3 of 4 )

Well

Depth to

Elevation, ft above ms 1

Water, ft Adjusted Casing Water Level

\begin{tabular}{|c|c|c|c|}
\hline $\begin{array}{l}199-N-66[N] \\
199-N-67 \\
199-N-08 S\end{array}$ & $\begin{array}{l}73.50 \\
67.78 \\
14.76\end{array}$ & $\begin{array}{l}465.25 \\
458.46 \\
404.57\end{array}$ & $\begin{array}{l}391.75 \\
390.68 \\
389.81\end{array}$ \\
\hline $\begin{array}{l}699-60-32 \\
699-60-60 \\
699-61-37 \\
699-61-41 \\
699-61-55 B\end{array}$ & $\begin{array}{r}62.79 \\
108.95 \\
60.89 \\
32.65 \\
52.08\end{array}$ & $\begin{array}{l}425.30 \\
512.03 \\
442.94 \\
428.92 \\
455.40\end{array}$ & $\begin{array}{l}362.52 \\
403.08 \\
382.05 \\
396.28 \\
403.32\end{array}$ \\
\hline $\begin{array}{l}699-61-57 \\
699-61-62 \\
699-61-66 \\
699-62-31 \\
699-62-43 A\end{array}$ & $\begin{array}{r}38.30 \\
94.48 \\
119.93 \\
71.65 \\
35.67\end{array}$ & $\begin{array}{l}441.85 \\
497.51 \\
522.18 \\
434.12 \\
432.30\end{array}$ & $\begin{array}{l}403.55 \\
403.03 \\
402.25 \\
362.47 \\
396.64\end{array}$ \\
\hline $\begin{array}{l}699-63-25 A \\
699-63-51 \\
699-63-58 \\
699-63-90 \\
699-64-62\end{array}$ & $\begin{array}{r}32.96 \\
24.09 \\
90.06 \\
109.39 \\
98.59\end{array}$ & $\begin{array}{l}395.15 \\
424.54 \\
491.90 \\
509.73 \\
500.25\end{array}$ & $\begin{array}{l}362.19 \\
400.45 \\
401.84 \\
400.34 \\
401.66\end{array}$ \\
\hline $\begin{array}{l}699.65-50 \\
699-65-59 A \\
699-65-72 \\
699-65-83 \\
699-65-95\end{array}$ & $\begin{array}{r}66.59 \\
105.43 \\
139.89 \\
85.61 \\
50.38\end{array}$ & $\begin{array}{l}467.06 \\
506.96 \\
540.28 \\
485.63 \\
452.26\end{array}$ & $\begin{array}{l}400.47 \\
401.53 \\
400.39 \\
400.03 \\
401.88\end{array}$ \\
\hline $\begin{array}{l}699-66-103 \\
699-66-23 \\
699-66-38 \\
699-66-39 \\
699-66-58\end{array}$ & $\begin{array}{r}61.08 \\
24.00 \\
33.44 \\
47.03 \\
101.96\end{array}$ & $\begin{array}{l}463.01 \\
389.01 \\
436.24 \\
453.78 \\
503.33\end{array}$ & $\begin{array}{l}401.93 \\
365.01 \\
402.80 \\
406.75 \\
401.37\end{array}$ \\
\hline $\begin{array}{l}699-66-64 \\
699-66-91 \\
699-67-51 \\
699-67-86 \\
699-67-98\end{array}$ & $\begin{array}{r}104.78 \\
66.27 \\
124.00 \\
72.57 \\
52.96\end{array}$ & $\begin{array}{l}505.92 \\
467.75 \\
524.59 \\
472.39 \\
455.47\end{array}$ & $\begin{array}{l}401.14 \\
401.48 \\
400.59 \\
399.82 \\
402.51\end{array}$ \\
\hline
\end{tabular}

LEGEND:

[N] Not located on maps to improve visuai clarity.

[Q] Questionable data - not included on map. 
Table 1. June 1990 Water Level Measurement Data, 100 Areas. (Sheet 4 of 4)

Well

Depth to

Elevation, $\mathrm{ft}$ above $\mathrm{ms} 1$ Water, ft Adjusted Casing Water Level

$\begin{array}{lrll}699-68-105 & 51.56 & 451.85 & 400.29 \\ 699-69-38 & 21.80 & 422.95 & 401.15 \\ 699-69-45 & 87.35 & 486.94 & 399.59 \\ 699-70-23 & 24.66 & 391.71 & 367.05 \\ 699-70-68 & 125.81 & 526.21 & 400.40 \\ 699-71-30 & 30.20 & 400.68 & \\ 699-71-52 & 123.12 & 523.04 & 370.48 \\ 699-71-77 & 73.24 & 472.28 & 399.92 \\ 699-72-73 & 83.72 & 482.57 & 399.04 \\ 699-72-88 & 34.28 & 437.37 & 398.85 \\ & & & 403.09 \\ 699-72-92 & 49.48 & 452.22 & 402.74 \\ 699-73-61 & 131.58 & 531.53 & 399.95 \\ 699-74-44 & 48.26 & 445.18 & 396.92 \\ 699-74-48 & 88.42 & 487.18 & 398.76 \\ 699-77-36 & 36.14 & 412.28 & 376.14 \\ & & & \\ 699-77-54 & 82.49 & 480.59 & 398.10 \\ 699-78-62 & 73.72 & 469.88 & 396.16 \\ 699-81-38 & 27.17 & 406.47 & 379.30 \\ 699-81-58 & 43.26 & 439.55 & 396.30 \\ 699-81-62 & 29.86 & 441.46 & 411.60 \\ 699-83-47 & 46.11 & 435.27 & \\ 699-86-42 & 25.21 & 409.92 & 389.16 \\ 699-87-55 & 70.03 & 458.63 & 384.74 \\ 699-88-41 & 33.78 & 416.04 & 388.60 \\ 699-89-35 & 21.53 & 397.46 & 382.26 \\ 699-90-45 & 37.43 & 422.15 & 375.93 \\ 699-91-37 & 47.19 & 422.93 & 384.72 \\ 699-92-49 & 48.12 & 432.00 & 375.74 \\ 699-96-49 & 36.33 & 419.29 & 383.88 \\ 699-97-43 & 42.30 & 421.81 & 382.96 \\ 699-99-42 & 31.96 & 412.88 & 379.51 \\ & & & 380.92\end{array}$

LEGEND:

[N] Not located on maps to improve visual clarity.

[Q] Questionable data - not included on map. 


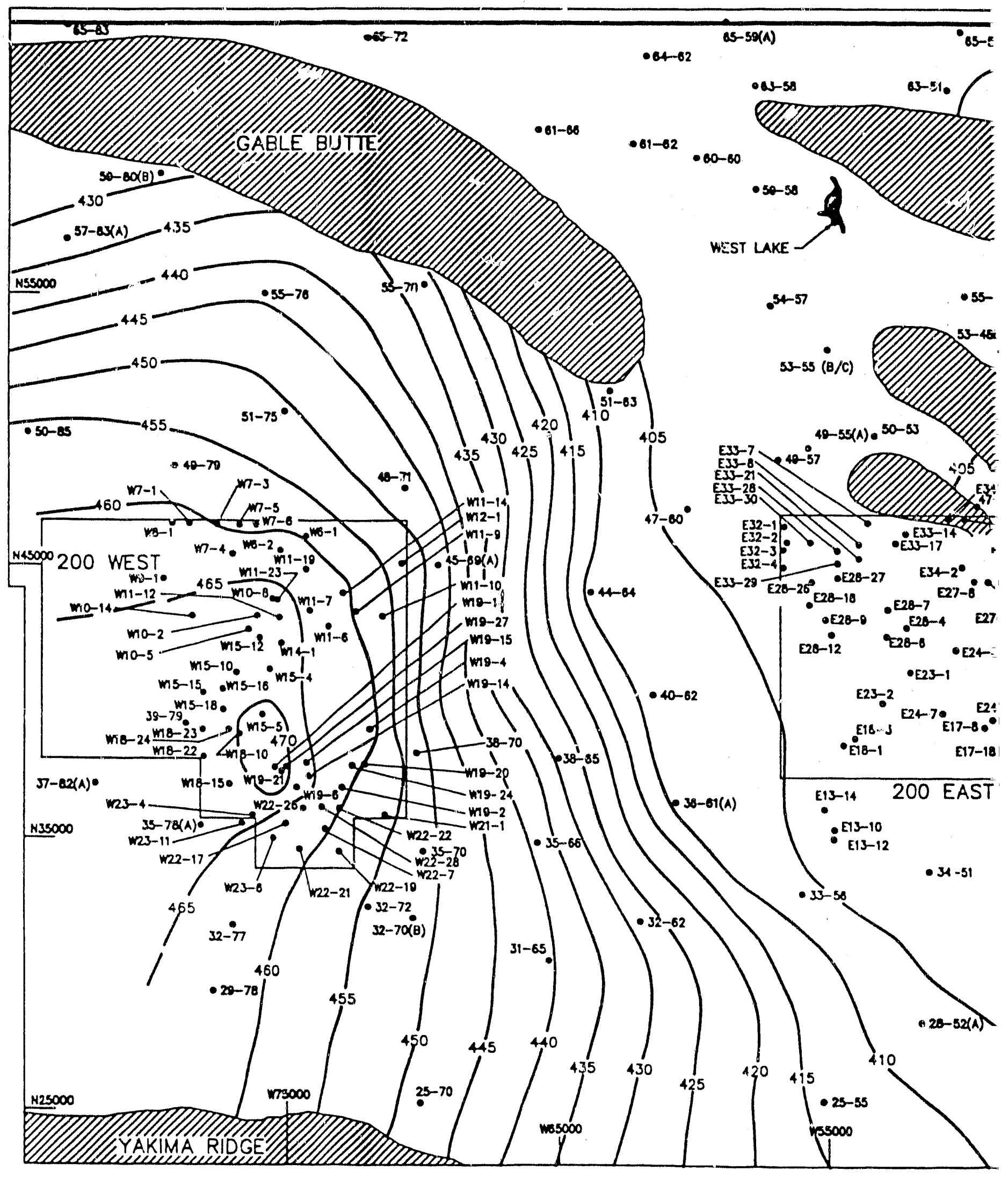




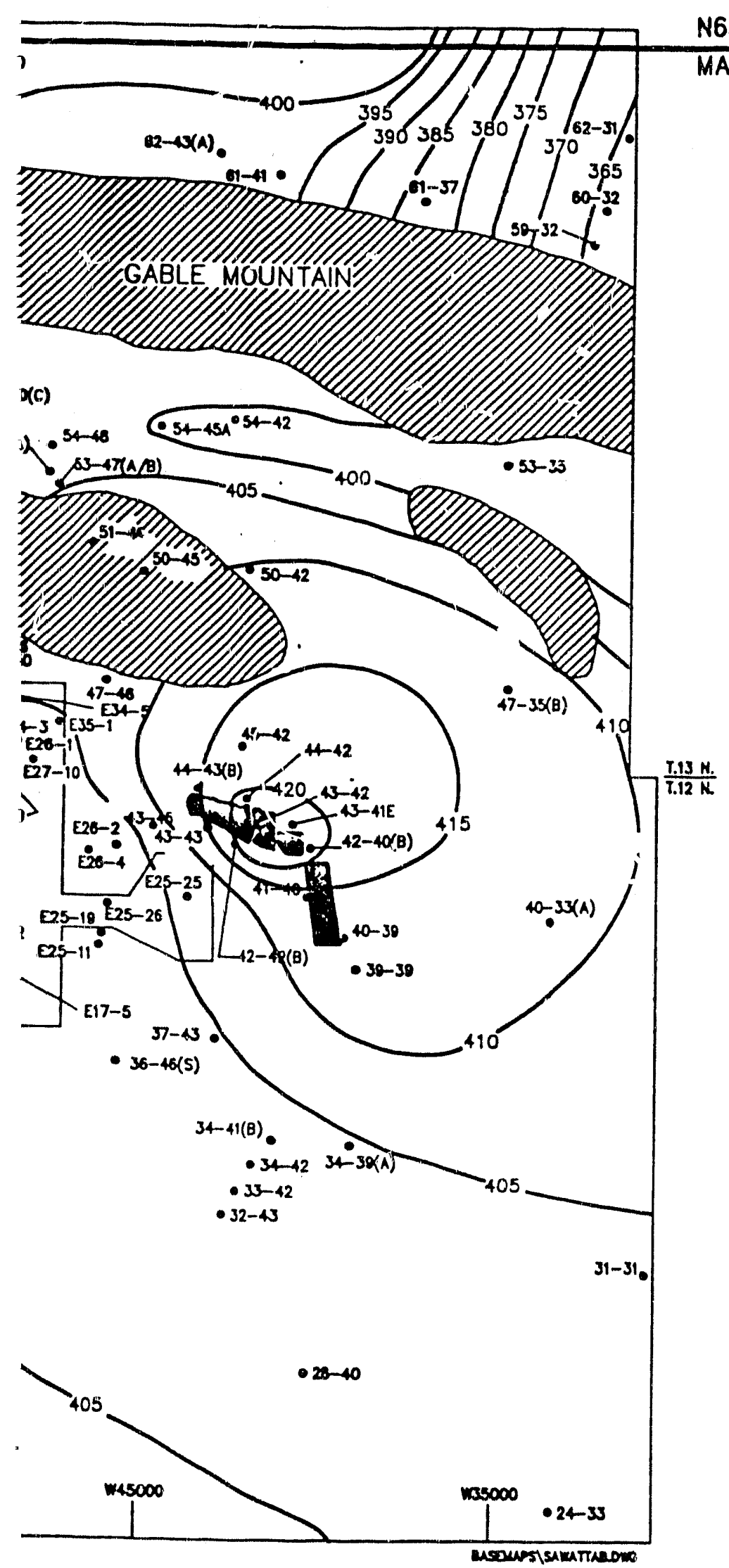

Figure 4

\section{Areas \\ Water Table Map June 1990}

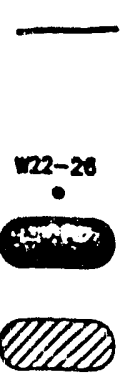

Woter toble contours in feet obove mean seo level

The 200 Areas water table map has been prepared by the Geosciences Group. Environmental Division. Westinghouse Hanford Company.

Note: To convert to metric, multiply elevation ( $\mathrm{ft}$ ) by 0.3048 to obtain elevation $(\mathrm{m})$.

Areas where the basalt surface is generally above the water table

Data points used to prepare map Ponds
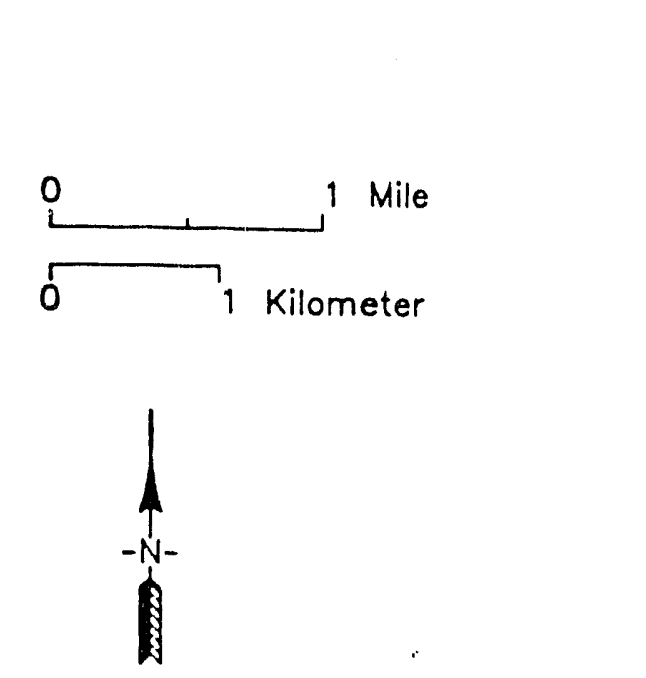


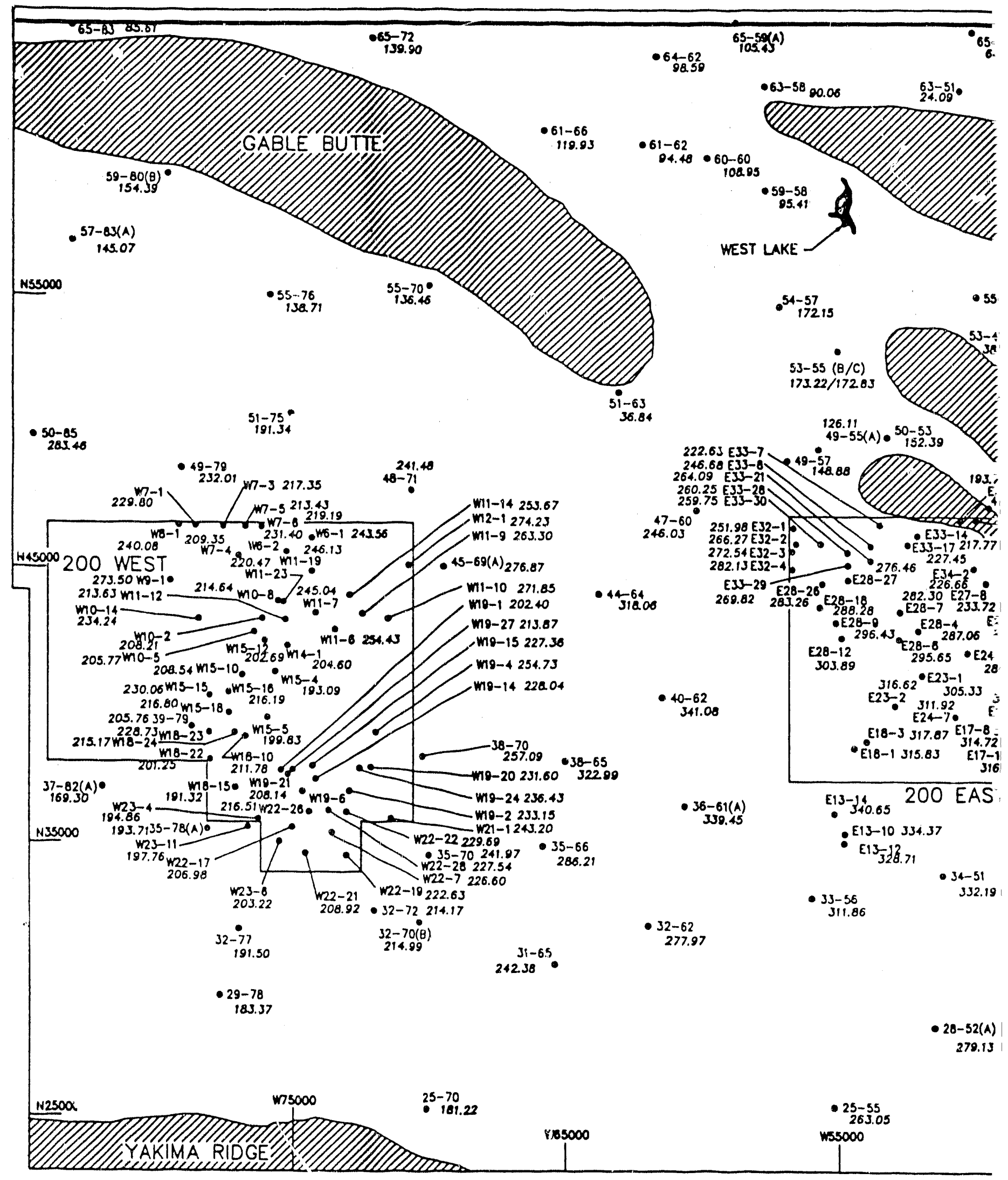




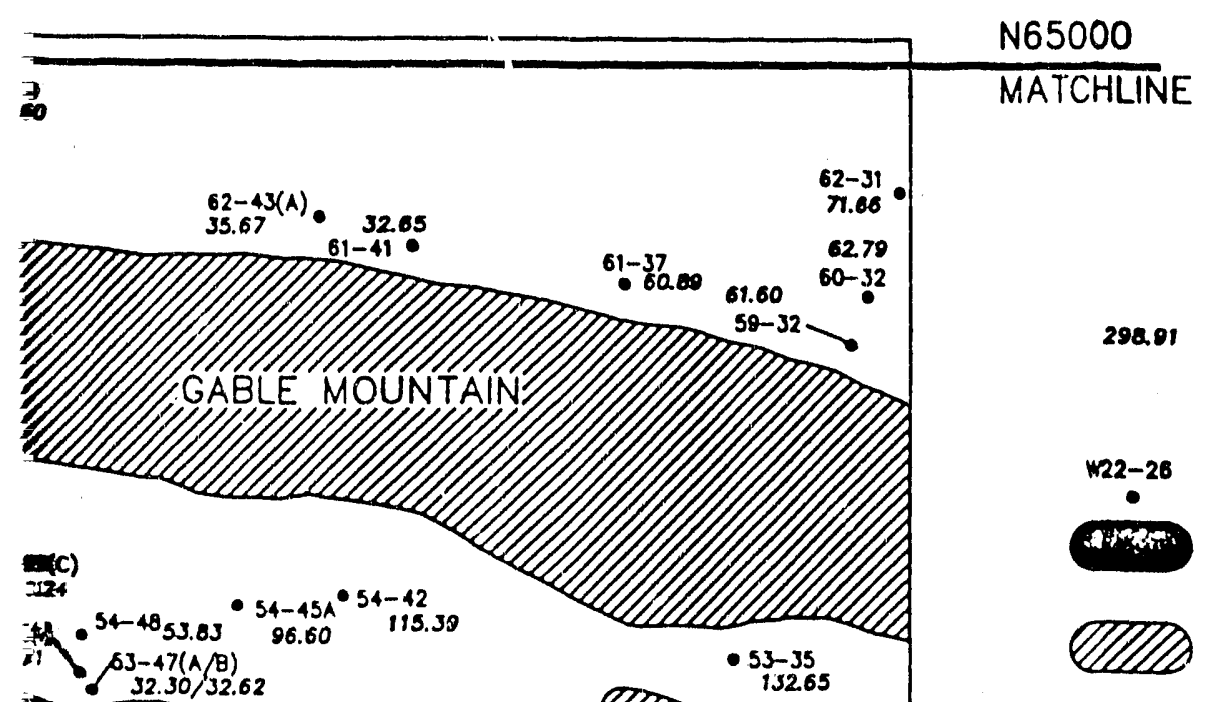

\section{,}

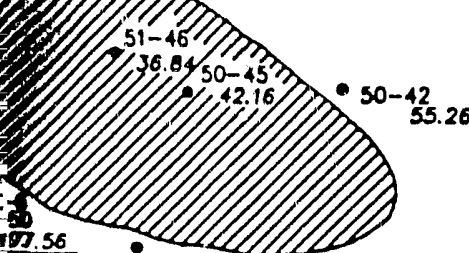

$=98+47-46$ 17.81 I0* 4 E35-1 193.6J 5026-1 212.37 क20 -10219.94 45-42 159.01 $230.61^{192.13}$ $44-43(8)+45-42+458.35$ 164.46 43-42 145.39 43-41E 129.5

- 43-43. 16422 -42-4O(B)

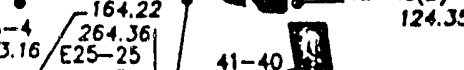
127 $272.96825-26$

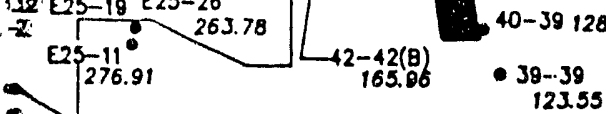
123.5

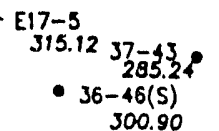

$34-41(B)$

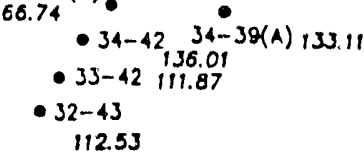

Figure 5

200 Areas

Depth-to-Water Map June 1990

Depth to water, as meosured from well reference mark (generally top of casing) to ground water surface.

Data points used to prepore map Ponds

Areas where the basalt surface is generally obove the woter table

The 200 Areos depth-to-water map has been prepored by the Geosciences Group. Environmental Division. Westinghouse Hanford Company.

Note: To convert to metric. multiply elevation (ft) by 0.3048 to obtain elevation $(\mathrm{m})$.

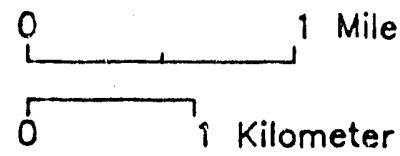




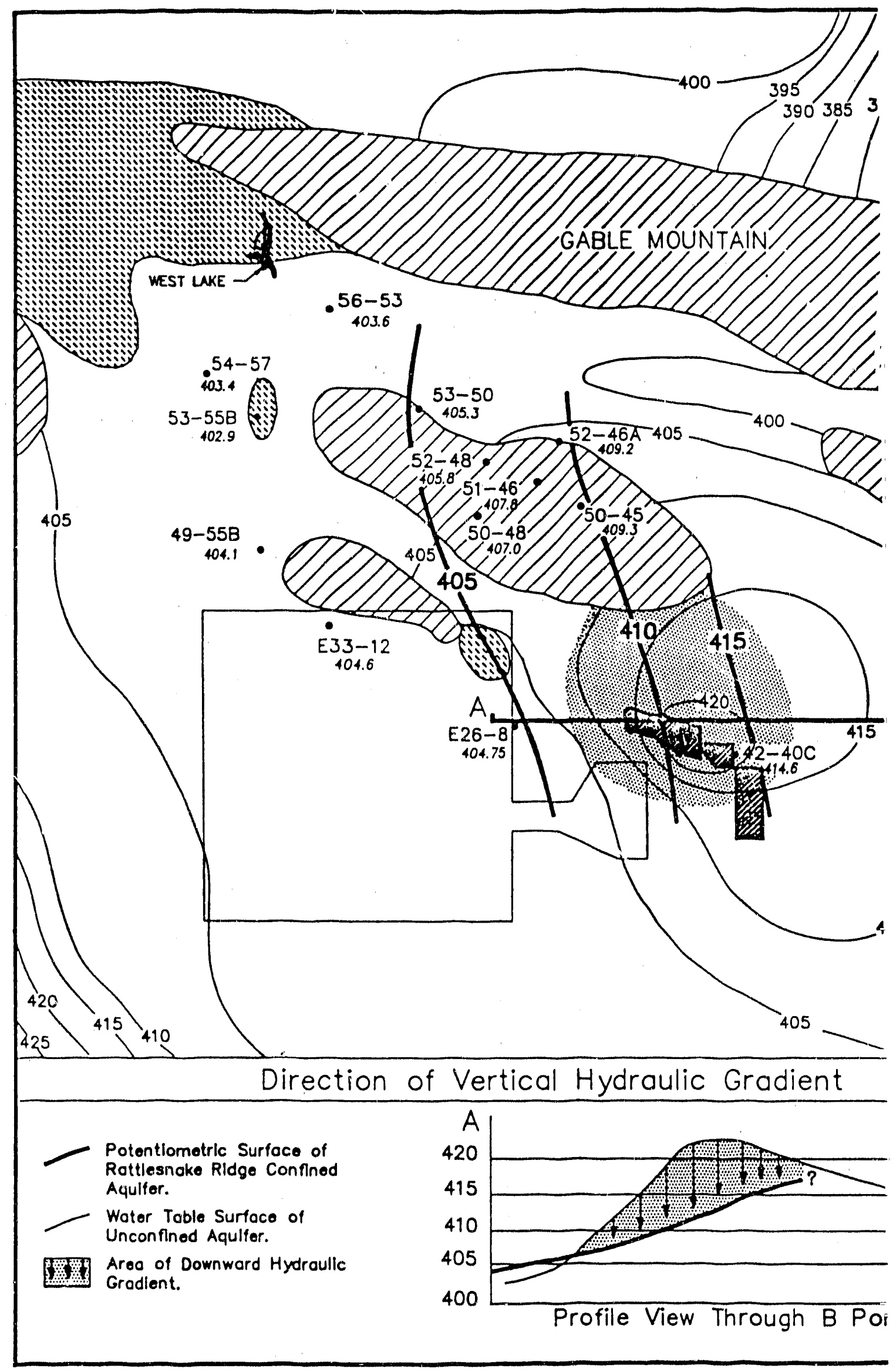


WHC -EP-0394-1

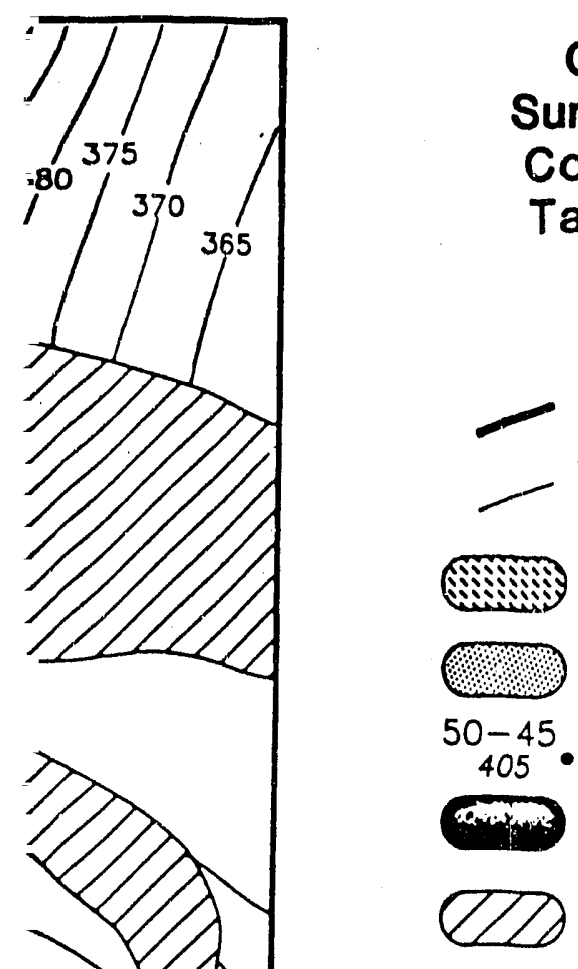

Figure 6

Comparison of Potentiometric

Surface of the Rattlesnake Ridge

Confined Aquifer with the Water Table of the Unconfined Aquifer

\section{June 1990}

Potentiometric surfoce of the Rottlesnoke Ridge confined aquifer in feet obove mean seo level

Water table contours in feet above mean sea level

Areas of complete erosion of the Elephant Mountain Member (from RHO-RE-ST-12)

Areo of downward hydroulic gradient

Wells in confined aquife- used to prepore mop

Pond

Generalized basalt above water table, os inferred $6 / 84$

The Rattlesnake Ridge aquifer, which is conflned by the Elephont Mountcih Member, is monitored quarterly in the eastern portion of the separations area. The June 1990 water-level measurements in 13 wells completed in the Rattlesnoke Ridge interbed were used to contour the potentiometric surface of the oquifer. Areal extent of downward hydroulic gradient from the unconfined aquifer to this confined aquifer is inferred from the water-table map and the contours of the potentiometric surfoce of the Rattlesnake Ridge. This area represents the zone in which downward flow might occur if a pothway is available due to complete erosion of the Elephant Mountain Member or sufficiently high hydroulic conductivity in the bosalt. A profile viow through the B Pond system shows the relationship between the unconfined water table and the potentiometric surface of the Rattlesnake Ridge conflned aqulfer.

The potentiometric surface of the Rattlesnake Ridge confined aquifer mop has been prepared by the Geoscienses group. Environmental Division. Westinghouse Honford Company.

Note: To convert to metric, multiply elevation ( $f t$ ) by 0.3048 to obtain elevation $(m)$.

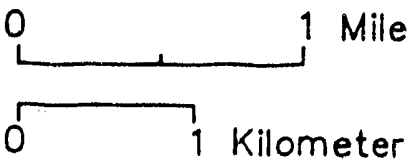


Table 2. June 1990 Water Level Measurement Data, 200 Areas. (Sheet 1 of 8 )

We11

Depth to

Elevation, ft above ms 1

Water, ft Adjusted Casing Water Level

$\begin{array}{llll}299-E 13-10 & 334.37 & 738.84 & 404.47 \\ 299-E 13-12 & 328.71 & 731.34 & 402.63 \\ 299-E 13-14 & 340.65 & 745.15 & 404.50 \\ 299-E 16-01[Q] & 287.76 & 696.44 & 408.68 \\ 299-E 17-18 & 316.65 & 720.65 & 404.00 \\ & & & \\ 299-E 17-05 & 315.12 & 718.69 & 403.57 \\ 299-E 17-08 & 314.72 & 718.38 & 403.66 \\ 299-E 18-01 & 315.83 & 720.24 & 404.42 \\ 299-E 18-03 & 317.87 & 722.04 & 404.17 \\ 299-E 23-01 & 305.33 & 709.65 & 404.32 \\ & & & \\ 299-E 23-02 & 316.62 & 720.64 & 404.02 \\ 299-E 24-18[N] & 315.2 .6 & 719.28 & 404.02 \\ 299-E 24-19[N] & 289.54 & 693.65 & 404.11 \\ 299-E 24-02 & 313.32 & 717.47 & 404.15 \\ 299-E 24-07 & 311.92 & 716.01 & 404.09 \\ 299-E 24-08 & 284.22 & 688.81 & \\ 299-E 25-11 & 276.91 & 681.28 & 404.59 \\ 299-E 25-19 & 272.96 & 677.20 & 404.38 \\ 299-E 25-25 & 264.36 & 669.42 & 404.24 \\ 299-E 25-26 & 263.78 & 668.52 & 405.06 \\ 299-E 25-27[N] & 271.40 & 676.08 & 404.74 \\ 299-E 25-37[N] & 268.97 & 673.29 & \\ 299-E 25-38[N] & 269.00 & 673.52 & 404.68 \\ 299-E 25-40[N] & 261.42 & 665.71 & 404.32 \\ 299-E 25-41[N] & 266.91 & 671.26 & 404.52 \\ 299-E 26-01 & 212.37 & 617.25 & 404.35 \\ 299-E 26-02 & 230.61 & 635.30 & 404.89 \\ 299-E 26-04 & 243.16 & 647.76 & 404.69 \\ 299-E 27-10 & 219.94 & 624.47 & 404.60 \\ 299-E 27-11[N] & 239.31 & 643.29 & 404.53 \\ 299-E 27-12[N] & 256.82 & 660.95 & 403.98 \\ 299-E 27-13[N] & 264.94 & 668.99 & 404.13 \\ & & & 404.05\end{array}$

LEGEND:

[N] Not located on maps to improve visual clarity.

[Q] Questionable data - not included on map.

[P] Located on piezometric surface map.

[S] Supplemental data wells not in operational network. 
Table. 2. June 1990 Water Level Measurement Data, 200 Areas. (Sheet 2 of 8 )

$\begin{array}{ll}\text { Well Elevation, ft above ms } 1 \\ \text { Water, ft } & \text { Adjusted Casing Water Level }\end{array}$

$\begin{array}{llll}299-E 27-14[N] & 254.18 & 658.34 & 404.16 \\ 299-E 27-15[N] & 248.88 & 652.67 & 403.79 \\ 299-E 27-08 & 233.72 & 637.83 & 404.11 \\ 299-E 27-09 & 224.97 & 629.21 & 404.24 \\ 299-E 28-12 & 303.89 & 708.60 & 404.71 \\ 299-E 28-17 & 303.32 & 708.56 & 405.24 \\ 299-E 28-18 & 288.28 & 692.58 & 404.30 \\ 299-E 28-26 & 283.26 & 687.26 & 404.00 \\ 299-E 28-27 & 276.46 & 680.37 & 403.91 \\ 299-E 28-04 & 287.06 & 691.55 & 404.49 \\ 299-E 28-06 & 295.65 & 700.11 & 404.46 \\ 299-E 28-07 & 282.30 & 685.91 & 403.61 \\ 299-E 28-09 & 296.43 & 700.77 & 404.34 \\ 299-E 32-01 & 251.98 & 656.17 & \\ 299-E 32-02 & 266.27 & 670.06 & 404.19 \\ 299-E 32-03 & 272.54 & 676.51 & 403.79 \\ 299-E 32-04 & 282.13 & 685.88 & 403.97 \\ 299-E 32-05[N] & 278.28 & 682.14 & 403.75 \\ 299-E 33-12[P] & 218.39 & 623.00 & 403.86 \\ 299-E 33-14 & 217.77 & 622.12 & 404.61 \\ 299-E 33-17 & 227.45 & 631.65 & 404.35 \\ 299-E 33-21 & 264.09 & 668.13 & 404.20 \\ 299-E 33-28 & 260.25 & 664.23 & 404.04 \\ 299-E 33-29 & 269.82 & 673.77 & 403.98 \\ 299-E 33-30 & 259.75 & 663.70 & 403.95 \\ 299-E 33-31[N] & 243.26 & 647.28 & 403.95 \\ 299-E 33-32[N] & 255.96 & 659.83 & 404.02 \\ 299-E 33-33[N] & 236.15 & 640.17 & 403.87 \\ 299-E 33-07 & 222.63 & 626.58 & 404.02 \\ 299-E 33-08 & 246.68 & 650.73 & 403.95 \\ 299-E 34-02 & 226.66 & 630.80 & 404.05 \\ 299-E 34-03 & 206.98 & 611.52 & 404.14 \\ & & & 404.54\end{array}$

\section{LEGEND:}

[N] Not located on maps to improve visual clarity.

[Q] Questionable data - not included on map.

[P] Located on piezometric surface map.

[S] Supplemental data wells not in operational network. 
Table 2. June 1990 Water Level Measurement Data, 200 Areas. (Sheet 3 of 8 )

Wepth to
Water, ft Adjusted Casing Water Level

$\begin{array}{llll}299-E 34-05 & 185.76 & 590.79 & 405.03 \\ 299-E 34-06 & 193.74 & 597.83 & 404.09 \\ 299-E 34-07[N] & 199.95 & 604.27 & 404.32 \\ 299-E 35-01 & 193.63 & 598.31 & 404.68 \\ 299-W 10-14 & 234.24 & 699.43 & 465.19 \\ 299-W 10-15[N] & 209.98 & 675.64 & 465.66 \\ & & & \\ 299-W 10-16[N] & 206.91 & 672.76 & 465.85 \\ 299-W 10-02 & 208.21 & 674.33 & 466.12 \\ 299-W 10-05 & 205.77 & 672.31 & 466.54 \\ 299-W 10-08 & 214.64 & 680.33 & 465.69 \\ 299-W 11-10 & 271.85 & 728.89 & 457.04 \\ & & & \\ 299-W 11-12 & 213.63 & 679.58 & 465.95 \\ 299-W 11-14 & 253.67 & 715.16 & 461.49 \\ 299-W 11-19 & 246.13 & 707.00 & 460.87 \\ 299-W 11-23 & 220.47 & 685.86 & 465.39 \\ 299-W 11-06 & 254.43 & 716.23 & 461.80 \\ 299-W 11-07 & 245.04 & 709.11 & \\ 299-W 11-09 & 263.30 & 722.94 & 464.07 \\ 299-W 12-01 & 274.23 & 726.46 & 459.64 \\ 299-W 14-01 & 204.60 & 665.83 & 452.23 \\ 299-W 14-09[0] & & & 461.23 \\ 299-W 15-10 & 208.54 & 676.00 & \\ 299-W 15-12 & 202.69 & 671.00 & 467.46 \\ 299-W 15-15 & 230.06 & 697.96 & 468.31 \\ 299-W 15-16 & 216.19 & 684.89 & 467.90 \\ 299-W 15-18 & 216.80 & 685.71 & 468.70 \\ 299-W 15-19[N] & 223.99 & 691.60 & 468.91 \\ 299-W 15-20 & 231.96 & 698.36 & 467.61 \\ 299-W 15-04 & 193.09 & 662.00 & 466.40 \\ 299-W 15-05 & 199.83 & 670.68 & 468.91 \\ 299-W 18-10 & 211.78 & 682.63 & 470.85 \\ & & & 470.85\end{array}$

[N] Not located on maps to improve visual clarity.

[Q] Questionable data - not included on map.

[P] Located on piezometric surface map.

[S] Supplemental data wells not in operational network. 
Table 2. June 1990 Water Level Measurement Data, 200 Areas. (Sheet 4 of 8 )

We11 Depth to

Elevation, ft above msl Water, ft Adjusted Casing Water Level

$\begin{array}{llll}299-W 18-15 & 191.32 & 660.76 & 469.44 \\ 299-W 18-22 & 201.25 & 668.49 & 467.24 \\ 299-W 18-23 & 288.73 & 696.81 & 468.08 \\ 299-W 18-24 & 215.17 & 684.35 & 469.18 \\ 299-W 18-26[N] & 231.01 & 699.05 & 468.04 \\ 299-W 19-01 & 202.40 & 673.77 & \\ 299-W 19-14 & 228.04 & 693.21 & 471.37 \\ 299-W 19-15 & 227.36 & 693.28 & 465.17 \\ 299-W 19-02 & 233.15 & 694.04 & 465.92 \\ 299-W 19-20 & 231.60 & 691.04 & 460.89 \\ 299-W 19-21 & 208.14 & 678.53 & 459.44 \\ 299-W 19-24 & 236.43 & 696.95 & 470.39 \\ 299-W 19-27 & 213.87 & 683.65 & 460.52 \\ 299-W 19-28[N] & 240.48 & 703.09 & 469.78 \\ 299-W 19-29[N] & 240.11 & 701.87 & 462.61 \\ 299-W 19-04 & 254.73 & & 461.76 \\ 299-W 19-06[Q] & 220.92 & 715.26 & \\ 299-W 21-01 & 243.20 & 700.00 & 460.53 \\ 299-W 22-17 & 206.98 & 699.26 & 479.08 \\ 299-W 22-19 & 222.63 & 671.62 & 456.06 \\ 299-W 22-21 & 208.92 & 681.26 & 464.64 \\ 299-W 22-22 & 229.69 & 670.00 & 458.63 \\ 299-W 22-26 & 216.51 & 690.05 & 461.08 \\ 299-W 22-28 & 227.54 & 680.30 & 460.36 \\ 299-W 22-07 & 226.60 & 689.00 & 463.79 \\ 299-W 23-11 & 197.76 & 687.41 & 461.46 \\ 299-W 23-04 & 194.86 & 664.14 & 460.81 \\ 299-W 23-06 & 203.22 & 662.82 & 466.38 \\ 299-W 6-01 & 243.56 & 667.00 & 467.96 \\ 299-W 6-02 & 231.40 & 702.53 & 463.78 \\ 299-W 7-01 & 229.80 & 692.45 & 458.97 \\ 299-W 7-03 & 217.35 & 690.71 & 461.05 \\ & & 676.14 & 460.91 \\ 29 & & 458.79\end{array}$

LEGEND:

[N] Not located on maps to improve visual clarity.

[Q] Questionable data - not included on map.

[P] Located on piezometric surface map.

[S] Supplemental data wells not in operational network. 
Table 2. June 1990 Water Level Measurement Data, 200 Areas. (Sheet 5 of 8 )

\begin{tabular}{|c|c|c|c|}
\hline Well & $\begin{array}{l}\text { Depth to } \\
\text { Water, ft }\end{array}$ & $\begin{array}{l}\text { Elevation, ft } \\
\text { Adjusted Casing }\end{array}$ & $\begin{array}{l}\text { above msl } \\
\text { Water Level }\end{array}$ \\
\hline $\begin{array}{l}29.9-W 7-04 \\
299-W 7-05 \\
299-W 7-06\end{array}$ & $\begin{array}{l}209.35 \\
213.43 \\
219.09\end{array}$ & $\begin{array}{l}671.69 \\
673.05 \\
678.64\end{array}$ & $\begin{array}{l}462.34 \\
459.62 \\
459.55\end{array}$ \\
\hline $\begin{array}{l}299-W 7-07[N] \\
299-W 7-08 \\
299-W 7-09 \\
299-W 8-01 \\
299-W 9-01\end{array}$ & $\begin{array}{l}215.27 \\
228.93 \\
230.79 \\
24.0 .08 \\
273.50\end{array}$ & $\begin{array}{l}674.94 \\
687.35 \\
692.11 \\
701.33 \\
737.73\end{array}$ & $\begin{array}{l}459.67 \\
458.42 \\
461.32 \\
461.25 \\
464.23\end{array}$ \\
\hline $\begin{array}{l}699-24-33 \\
699-25-55 \\
699-25-70 \\
699-28-40 \\
699-28-52 A\end{array}$ & $\begin{array}{l}120.78 \\
263.05 \\
181.22 \\
155.54 \\
279.13\end{array}$ & $\begin{array}{l}524.21 \\
676.55 \\
629.78 \\
559.44 \\
684.67\end{array}$ & $\begin{array}{l}403.43 \\
413.50 \\
448.56 \\
403.90 \\
405.54\end{array}$ \\
\hline $\begin{array}{l}699-29-78 \\
699-31-31[S] \\
699-31-65 \\
699-32-43 \\
699-32-62\end{array}$ & $\begin{array}{l}183.37 \\
125.90 \\
242.38 \\
112.53 \\
277.97\end{array}$ & $\begin{array}{l}647.05 \\
529.32 \\
683.09 \\
516.62 \\
707.09\end{array}$ & $\begin{array}{l}463.68 \\
403.42 \\
440.71 \\
404.09 \\
429.12\end{array}$ \\
\hline $\begin{array}{l}699-32-70 B \\
699-32-72 \\
699-32-77 \\
699-33-42 \\
699-33-56\end{array}$ & $\begin{array}{l}214.99 \\
214.17 \\
191.50 \\
111.87 \\
311.86\end{array}$ & $\begin{array}{l}666.68 \\
668.16 \\
653.74 \\
516.00 \\
717.03\end{array}$ & $\begin{array}{l}451.69 \\
453.99 \\
462.24 \\
404.13 \\
405.17\end{array}$ \\
\hline $\begin{array}{l}699-34-39 A \\
699-34-41 B[S] \\
699-34-42 \\
699-34-51 \\
699-35-66\end{array}$ & $\begin{array}{l}133.11 \\
166.74 \\
136.01 \\
332.19 \\
286.21\end{array}$ & $\begin{array}{l}537.07 \\
570.89 \\
540.20 \\
736.76 \\
725.65\end{array}$ & $\begin{array}{l}403.96 \\
404.15 \\
404.19 \\
404.57 \\
439.44\end{array}$ \\
\hline $\begin{array}{l}699-35-70 \\
699-35-78 A \\
699-36-46 S \\
699-36-61 A\end{array}$ & $\begin{array}{l}241.97 \\
193.71 \\
300.90 \\
339.45\end{array}$ & $\begin{array}{l}693.72 \\
660.65 \\
704.33 \\
748.11\end{array}$ & $\begin{array}{l}451.75 \\
466.94 \\
403.43 \\
409.66\end{array}$ \\
\hline
\end{tabular}

\section{LEGEND:}

[N] Not located on maps to improve visual clarity.

[Q] Questionable data - not included on map.

[P] Located on piezometric surface map.

[S] Supplemental data wells not in operational network. 
Table 2. June 1990 Water Level Measurement Data, 200 Areas. (Sheet 6 of 8 )

Well

Depth to

Elevation, ft above msl

Water, ft Adjusied Casing Water Level

$\begin{array}{lrll}699-37-43 & 285.24 & 690.17 & 404.93 \\ 699-37-82 A & 169.30 & 636.75 & 467.45 \\ 699-38-65 & 322.99 & 753.33 & 430.34 \\ 699-38-70 & 257.09 & 710.67 & 453.58 \\ 699-39-39 & 123.55 & 536.65 & 413.10 \\ 699-39-79 & 205.76 & 673.52 & 467.76 \\ & & & \\ 699-40-33 A & 106.79 & 518.05 & 411.26 \\ 699-40-39 & 128.13 & 541.84 & 413.71 \\ 699-40-62[S] & 341.08 & 747.78 & 406.70 \\ 699-41-40 & 129.62 & 545.94 & 416.32 \\ 699-42-40 B & 124.35 & 546.46 & 422.11 \\ & & & \\ 699-42-40 C[P] & 131.57 & 546.16 & 414.59 \\ 699-42-42 B & 165.96 & 583.23 & 417.27 \\ 699-43-41 E & 129.58 & 550.86 & 421.28 \\ 699-43-41 F & 129.59 & 551.01 & 421.42 \\ 699-43-42[S] & 145.39 & 566.36 & 420.97 \\ 699-43-43 & 164.22 & 579.37 & \\ 699-43-45 & 192.13 & 597.68 & 415.15 \\ 699-44-42 & 158.33 & 579.22 & 405.55 \\ 699-44-43 B & 164.46 & 580.12 & 420.89 \\ 699-44-64 & 318.06 & 725.60 & 415.66 \\ 699-45-42 & 159.81 & 577.33 & 407.54 \\ 699-45-69 A[S] & 276.89 & 725.46 & 417.52 \\ 699-47-35 B & 62.50 & 476.65 & 448.57 \\ 699-47-46 A & 174.81 & 580.14 & 414.15 \\ 699-47-50 & 174.56 & 583.87 & 405.33 \\ 699-47-60 & 246.03 & 649.84 & 406.31 \\ 699-48-71 & 241.48 & 688.15 & 403.81 \\ 699-49-55 A & 126.11 & 530.14 & 446.67 \\ 699-49-55 B[P] & 126.28 & 530.33 & 404.03 \\ 699-49-57 & 148.88 & 552.81 & 404.05 \\ & & & 403.93\end{array}$

LEGEND:

[N] Not located on maps to improve visual clarity.

[Q] Questionable data - not included on map.

[P] Located on piezometric surface map.

[S] Supplemental data wells not in operational network. 
Table 2. June 1990 Water Level Measurement Data, 200 Areas. (Sheet 7 of 8 )

We11. Elevation, ft above ms 1
Water, ft
Wajusted Cacing Water Level

\begin{tabular}{|c|c|c|c|}
\hline $\begin{array}{l}699-49-79 \\
699-50-42 \\
699-50-45 \\
699-50-53 \\
699-50-85\end{array}$ & $\begin{array}{r}232.01 \\
55.26 \\
42.16 \\
152.39 \\
283.46\end{array}$ & $\begin{array}{l}689.20 \\
466.84 \\
451.41 \\
556.30 \\
739.35\end{array}$ & $\begin{array}{l}457.19 \\
411.58 \\
409.25 \\
403.91 \\
455.89\end{array}$ \\
\hline $\begin{array}{l}699-51-46 \\
699-51-63 \\
699-51-75 \\
699-52-46 A[P] \\
699-52-48[P]\end{array}$ & $\begin{array}{r}36.84 \\
165.84 \\
191.34 \\
46.38 \\
60.24\end{array}$ & $\begin{array}{l}444.63 \\
571.84 \\
641.51 \\
455.61 \\
466.06\end{array}$ & $\begin{array}{l}407.79 \\
406.00 \\
450.17 \\
409.23 \\
405.82\end{array}$ \\
\hline $\begin{array}{l}699-53-35 \\
699-53-47 A \\
699-53-47 B \\
699-53-48 A \\
699-53-48 B\end{array}$ & $\begin{array}{r}132.65 \\
32.30 \\
32.62 \\
38.71 \\
38.23\end{array}$ & $\begin{array}{l}530.66 \\
438.28 \\
438.58 \\
442.45 \\
442.71\end{array}$ & $\begin{array}{l}398.34 \\
405.98 \\
405.96 \\
403.74 \\
404.48\end{array}$ \\
\hline $\begin{array}{l}699-53-50[P] \\
699-53-55 B \\
699-53-55 C \\
699-54-42 \\
699-54-45 A\end{array}$ & $\begin{array}{r}38.93 \\
173.22 \\
172.83 \\
115.39 \\
96.60\end{array}$ & $\begin{array}{l}444.21 \\
576.16 \\
576.08 \\
511.49 \\
494.29\end{array}$ & $\begin{array}{l}405.29 \\
402.94 \\
403.25 \\
396.10 \\
397.69\end{array}$ \\
\hline $\begin{array}{l}699-54-48 \\
699-54-57 \\
699-55-40[Q] \\
699-55-50 C \\
699-55-70\end{array}$ & $\begin{array}{r}53.83 \\
172.15 \\
133.10 \\
41.24 \\
136.46\end{array}$ & $\begin{array}{l}457.02 \\
575.58 \\
543.13 \\
444.43 \\
569.03\end{array}$ & $\begin{array}{l}403.19 \\
403.43 \\
410.03 \\
403.19 \\
432.57\end{array}$ \\
\hline $\begin{array}{l}699-55-76 \\
699-56-53[P] \\
699-57-83 A[S] \\
699-59-32 \\
699-59-58\end{array}$ & $\begin{array}{r}138.71 \\
30.72 \\
145.07 \\
61.60 \\
95.41\end{array}$ & $\begin{array}{l}583.24 \\
434.34 \\
577.96 \\
424.29 \\
497.77\end{array}$ & $\begin{array}{l}444.53 \\
403.62 \\
432.89 \\
362.69 \\
402.36\end{array}$ \\
\hline $\begin{array}{l}699-59-80 B \\
699-60-32\end{array}$ & $\begin{array}{r}154.39 \\
62.79\end{array}$ & $\begin{array}{l}583.25 \\
425.30\end{array}$ & $\begin{array}{l}428.86 \\
362.51\end{array}$ \\
\hline
\end{tabular}

LEGEND:

[N] Not located on maps to improve visual clarity.

[Q] Questionable data - not included on map.

[P] Located on piezometric surface map.

[S] Supplemental data wells not in operationai network. 
Table 2. June 1990 Water Level Measurement Data, 200 Areas. (Sheet 8 of 8 )

$\begin{array}{crcc}\text { Well } & \begin{array}{c}\text { Elevth to } \\ \text { Water, ft }\end{array} & \begin{array}{c}\text { Adjusted Casing } \\ \text { Ad Water Level }\end{array} & \\ & & & \\ 699-60-60 & 108.95 & 512.03 & 403.08 \\ 699-61-37 & 60.89 & 442.94 & 382.05 \\ 699-61-41 & 32.65 & 428.92 & 396.27 \\ 699-61-62 & 94.48 & 497.51 & 403.03 \\ 699-61-66 & 119.93 & 522.18 & 402.25 \\ 699-62-31 & 71.66 & 434.12 & 362.46 \\ 699-62-43 A & 35.67 & 432.30 & 396.63 \\ 699-63-51 & 24.09 & 424.54 & 400.45 \\ 699-63-53 & 90.06 & 491.90 & 401.84 \\ 699-64-62 & 98.59 & 500.25 & 401.66 \\ 699-65-50 & 66.60 & 467.06 & 400.46 \\ 699-65-59.4 & 105.43 & 506.96 & 401.53 \\ 699-65-72 & 139.90 & 540.28 & 400.38 \\ 699-65-83 & 85.61 & 485.63 & 400.02\end{array}$

LEGEND:

[N] Not located on maps to improve visual clarity.

[Q] Questionable data - not included on map.

[P] Located on piezometric surface map.

[S] Supplemental data wells not in operational network. 

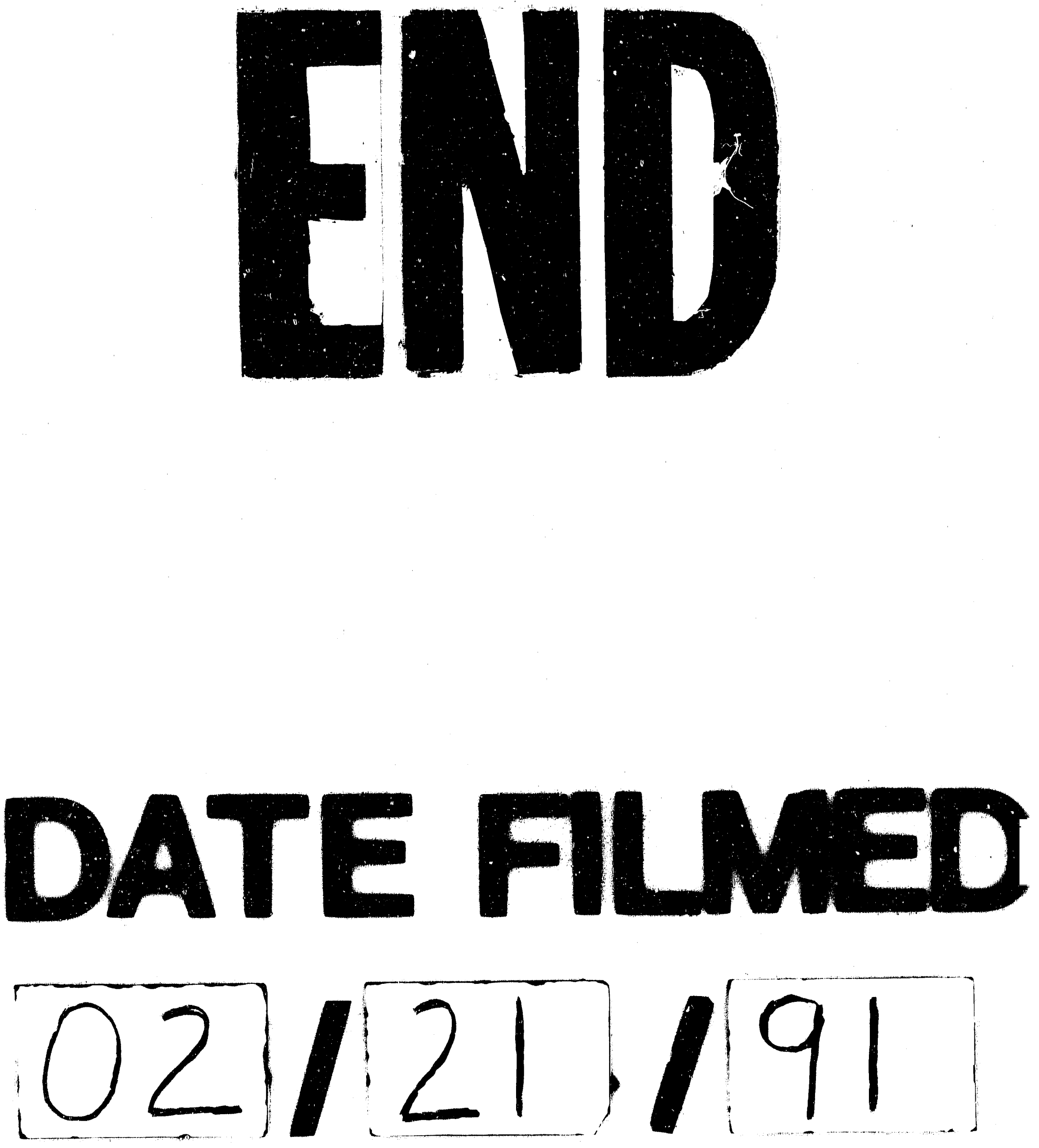
$14, \quad, \quad$ 